\title{
Inclusión de la perspectiva de interseccionalidad y la justicia reproductiva en el marco del cambio climático desde la bioética
}

\section{Intersectionality and Reproductive Justice in the Context of Climate Change from Bioethics}

\author{
Ana Violeta Trevizo \\ Universidad Nacional Autónoma de México | México \\ Contacto: violeta.trevizo@gmail.com
}

\section{Resumen}

Este trabajo propone la inclusión de la perspectiva de interseccionalidad y la justicia reproductiva en el marco del cambio climático y el abordaje de las políticas públicas de mitigación desde la perspectiva bioética. Plantea la vulnerabilidad que viven los grupos marginados y las inequidades interseccionales como el eje central en la falta de autonomía y libertad reproductiva; y cómo esas inequidades se exacerban con el cambio climático, afectando mayormente a mujeres pobres, indígenas, afrodescendientes, y migrantes. Se propone el principio de igualdad de trato y de oportunidades entre mujeres y hombres, el empoderamiento económico y educativo de las mujeres, su participación e integración en las decisiones políticas sobre el cambio climático, y la inclusión de la transversalidad de género en la discusión y análisis de la crisis climática. Llamamos a una conciencia ética bajo el eje de la biofilia o liga básica con el todo de la vida y el proceso de evolución propuesta por O. Wilson y seguida por L. Sagols.

Palabras clave: interseccionalidad, justicia reproductiva, cambio climático, vulnerabilidad mujeres

\section{Abstract:}

This work proposes the inclusion of the perspective of intersectionality and reproductive justice in the context of climate change and the approach of mitigation 
policies from a bioethical perspective. It poses the vulnerability experienced by marginalized groups and intersectional inequalities as the central axle of the lack of autonomy and reproductive freedom. And how these inequities are exacerbated by climate change, affecting mostly poor women, indigenous, African descent, and migrants. It proposes the principle of equal treatment and opportunities between women and men, the economic and educational empowerment of women, their participation and integration in political decisions on climate change, and the inclusion of gender mainstreaming in the discussion and analysis of the climate crisis. We call for an ethical conscience under the axis of biophilia or a basic link with the whole of life and the process of evolution proposed by $\mathrm{O}$. Wilson and followed by L. Sagols.

Keywords: intersectionality, reproductive justice, climate change, vulnerability, women

El cambio climático tendrá consecuencias devastadoras para las personas en situación de pobreza. Incluso en el mejor de los casos, cientos de millones se enfrentarán a la inseguridad alimentaria, la migración forzada, las enfermedades y la muerte. El cambio climático amenaza el futuro de los derechos humanos y corre el riesgo de deshacer los últimos cincuenta años de progreso en materia de desarrollo, salud mundial y reducción de la pobreza.

Philip Alston ${ }^{1}$

\section{Introducción}

Fue a partir de 1979 en la Primera Conferencia Mundial sobre el Clima en Ginebra que se consideró el cambio climático como una amenaza real para el planeta. La conferencia adoptó una declaración que exhortaba a los gobiernos a prever y evitar los posibles cambios en el clima provocados por el ser humano. A pesar de la evidente manifestación del cambio climático con el aumento del nivel del mar,

\footnotetext{
${ }^{1}$ Relator especial de la ONU sobre la pobreza extrema de junio de 2014 - abril de 2020. Informe sobre Cambio climático y pobreza. Consejo de Derechos Humanos, 41er período de sesiones. 24 de junio a 12 de julio de 2019. A/HRC/41/39.
} 
de la temperatura de la superficie terrestre, de la temperatura oceánica, la disminución de la extensión de nieves y hielos, el cambio en los patrones de precipitación, el aumento de los eventos climáticos extremos, la acidificación oceánica (de Vengoechea, 2012: 5-6), y las repercusiones de estos cambios en los seres vivos que habitan la Tierra, existen hoy en día agentes de poder que impulsan la negación del cambio climático, particularmente en Estados Unidos. Entre ellos, encontramos corporaciones y grupos de expertos conservadores y libertarios y la industria del carbón con el interés propio de evitar cualquier plan gubernamental para reducir las emisiones de carbono (Collomb, 2014: 1-2).

El cambio climático y los desastres naturales tienen un efecto desproporcionado, principalmente en mujeres y niños, al igual que los conflictos bélicos y la migración (Naciones Unidas, 2018: 31-34). Las actitudes culturales, las políticas públicas, la legislación, las instituciones tienen un gran impacto ambiental y social. En este trabajo se propone la inclusión de la perspectiva de interseccionalidad y la justicia reproductiva en el marco del cambio climático, así como su incorporación en la toma de decisiones y en las políticas públicas con el fin de proteger a las poblaciones más vulneradas y minimizar el dańo en su sustento y forma de vida. Además, se busca tratar de prevenir los daños a la salud y proteger los derechos fundamentales de las mujeres y las niñas porque son las más afectadas por el cambio climático y las desigualdades interseccionales, ya que cuentan con menor acceso a la educación y oportunidades laborales; viven en malnutrición, no tienen acceso a un sistema de salud, corren el riesgo de embarazos adolescentes o jóvenes, ${ }^{2}$ partos precarios $^{3}$ y el cuidado de hijos con mayor riesgo de enfermar desde la etapa de gestación. Todo ello, debido a las condiciones ocasionadas por la crisis ambiental, incluyendo la contaminación del aire, de los suelos y del agua.

Según el documento "Género y Cambio Climático" del programa de las Naciones Unidas para el Desarrollo, 80 \% de las personas desplazadas por el cambio

2 En México, el INEGI registró en 2017 que un total de 390089 mujeres menores de 20 ańos fueron madres; de ellas, 9748 eran niñas menores a 15 años y 380 341, adolescentes entre 15 y 19 años. El embarazo adolescente es un fenómeno multifactorial que requiere políticas públicas efectivas e integrales dirigidas a promover y garantizar el acceso a métodos anticonceptivos amigables y sin discriminación, a prevenir y atender la violencia sexual, a impartir educación sexual integral, garantizar el acceso a la interrupción legal del embarazo y a una atención médica especializada, entre otros (Grupo de Información en Reproducción Elegida, 2018: 16).

${ }^{3}$ Más de 300000 mujeres mueren anualmente por causas relacionadas con el embarazo en países desarrollados y en desarrollo. "Esto no debería estar pasando cuando ya tenemos las tecnologías para evitarlo, si queremos alcanzar los Objetivos para el 2030, tenemos que triplicar nuestros esfuerzos", declaró ante la prensa, Phumzile Mlambo-Ngcuka, directora ejecutiva de ONU Mujeres (ONU Mujeres, 2014). 
climático son mujeres (United Nations Development Programme, 2016: 5). Además, padecen los efectos del aumento de temperatura que puede causar estrés por calor y afectar la salud de sus hijos, de los enfermos y de los ancianos normalmente bajo su cuidado; asimismo, se enfrentan al peligro de contraer enfermedades infecciosas por las condiciones de pobreza en las que viven en zonas rurales y urbanas. ${ }^{4}$ La Organización Mundial de la Salud estima que aproximadamente 250000 muertes anuales entre 2030 y 2050 podrían deberse al cambio climático (Haines y Ebi, 2019: 266).

Las mujeres enfrentan de manera distinta la crisis climática. Esto dependerá de su contexto geográfico, étnico, social y económico. Por ejemplo, después del paso del huracán Katrina en el año 2005, en Nuevo Orleans, las mujeres afroamericanas en condición de pobreza fueron predominantemente las más afectadas (Haines y Ebi, 2019: 266), ya que vivían en situación de pobreza desde mucho antes del huracán. Además, no pudieron evacuar debido a la falta de medios para hacerlo. Un tercio de los residentes de Nueva Orleans en aquel tiempo no contaba con un auto y el plan oficial de evacuación contemplaba que lo hicieran por ese medio. Si las mujeres tenían un vehículo, no contaban con los medios económicos para pagar el combustible o una habitación de hotel, ya que el desastre ocurrió a final de mes, es decir, cuando ya habían gastado su salario. Una muestra notable de racismo fue que los autos conducidos por personas blancas pudieron evacuar, a diferencia de las mujeres afroamericanas que trataron de hacerlo a pie a través de los puentes, y no les fue permitida la evacuación. Otro obstáculo para la evacuación fue que ellas ya contaban con algún problema de salud o discapacidad o contaban con algún familiar con dichos problemas bajo su cuidado, volviéndolas más vulnerables a las condiciones extremas después de la tormenta, cuando no había alimentos, agua, electricidad, atención médica y otras necesidades básicas (Butterbaugh, 2005: 17). Las mujeres alrededor del mundo se enfrentan de manera diferente a la crisis climática. Ello dependerá de las desigualdades interseccionales que viven en relación con su condición social y económica, su diversidad funcional o discapacidad, su edad, su ciclo de vida familiar, su origen o migración, el problema de racialización, su religión, creencias, y otros ejes de desigualdad que cambian y se intersecan de acuerdo con su contexto. Es decir, no por ser mujer se vive el cambio climático de igual forma, por lo que la inclusión de la perspectiva de interseccionalidad es indispensable en la discusión ante la crisis climática.

\footnotetext{
${ }^{4}$ Como dengue, zika, chikungunya, enfermedad de Chagas, fiebre amarilla, paludismo, entre otras, que pueden ser grave e incluso causar la muerte.
} 
La Cuarta Conferencia Mundial sobre la Mujer, celebrada en Beijing en 1995, marcó un importante punto de inflexión para la agenda mundial de igualdad de género. La Declaración y Plataforma de Acción de Beijing, adoptada de forma unánime por 189 países, constituye un programa en favor del empoderamiento de la mujer y en su elaboración se tuvo en cuenta el documento clave de política mundial sobre igualdad de género. Dicha declaración, establece una serie de objetivos estratégicos y medidas para el progreso de las mujeres y el logro de la igualdad de género en 12 esferas cruciales que abarcan los siguientes temas: 1) la mujer y la pobreza, 2) la educación y capacitación de la mujer, 3) la mujer y la salud, 4) la violencia contra la mujer, 5) la mujer y los conflictos armados, 6) la mujer y la economía, 7) la mujer en el ejercicio del poder y la adopción de decisiones, 8) los mecanismos institucionales para el adelanto de la mujer, 9) los derechos humanos de la mujer, 10) la mujer y los medios de difusión, 11) la mujer y el medio ambiente y 12) las nińas (ONU Mujeres, 2014).

\section{Decisiones reproductivas ante la crisis climática}

Garantizar el acceso universal a la salud reproductiva y sexual, y otorgar a las mujeres derechos igualitarios en el acceso a recursos económicos, como tierras y propiedades, son condiciones fundamentales para afrontar la crisis climática y para que ellas tomen decisiones en beneficio de sí mismas y de su comunidad. Hoy más mujeres ocupan cargos públicos, sin embargo, es necesario que muchas más mujeres se conviertan en líderes (ONU Mujeres, 2014), que formen parte de los consejos, paneles y organismos nacionales e internacionales, y que de esta forma cuenten con poder político para participar en la toma decisiones e influyan en la elaboración de políticas preventivas que fomenten un desarrollo de vida más equilibrado para ellas frente a la crisis climática. Lograr justicia, equidad y autonomía para las mujeres más marginadas tendría que ser uno de los objetivos principales en la discusión sobre el cambio climático.

No podemos entender la experiencia de la fertilidad, de la reproducción y la maternidad, separada de nuestro entendimiento de la comunidad, del contexto social y ambiental en la que ocurre. La salud reproductiva, según la Declaración y Plataforma de Acción de Beijing antes mencionada, consiste en un estado general de bienestar físico, mental y social, y no en la mera ausencia de enfermedades o dolencias, en todos los aspectos relacionados con el sistema reproductivo, sus funciones y procesos. En consecuencia, la salud reproductiva entraña la capacidad de disfrutar de una vida sexual satisfactoria sin riesgos, la capacidad de procrear y la 
libertad para decidir hacerlo o no, ya sea de manera natural o a través de la reproducción médicamente asistida. Esta última condición lleva implícito el derecho que tenemos todos a obtener información de los métodos para la regulación de la fecundidad, acceso a métodos seguros, eficaces, asequibles y aceptables, el derecho a recibir servicios adecuados de atención de salud que permitan embarazos y partos sin riesgos, y den a las parejas las máximas posibilidades de tener hijos sanos $(\mathrm{Na}-$ ciones Unidas, 2014: 34-62). Estas condiciones se ven reducidas por desigualdades en las que, además de las relaciones género-ambiente, interviene la pobreza, una carga específica de trabajo en las labores cotidianas, la exposición a condiciones tóxicas del ambiente y las consecuencias de la crisis climática, como sequías, hambrunas o desastres naturales en los que las mujeres están lejos de contar con los mínimos indispensables para tomar decisiones reproductivas equilibradas, seguras para ellas, su comunidad y los otros seres vivos en el planeta.

La justicia reproductiva y la interseccionalidad deben colocarse en el centro de la agenda, en el marco de las políticas públicas sobre el cambio climático, al igual que en los acuerdos, declaraciones, convenciones y leyes internacionales, regionales y subregionales. Cuando hablamos de justicia reproductiva, nos referimos al conjunto de circunstancias sociales, políticas y económicas que permiten a las mujeres tener el poder y la autodeterminación sobre su destino reproductivo. Asimismo, nos referimos al acceso a los medios para llevarla a cabo, tanto para planificar un embarazo, como para interrumpirlo, o buscarlo de manera natural o por medio de tecnologías de reproducción asistida.

En cuanto a la interseccionalidad, ésta es compleja porque tiene que ver con localizaciones de las relaciones de poder, con colonizaciones, con una distribución jerarquizada de privilegios y desventajas. Una de las características del movimiento feminista actual es abordar la intersección de las opresiones. Las mujeres somos de clases sociales distintas, de culturas y religiones diferentes; podemos estar marcadas por la raza o la etnicidad, podemos vivir en países desarrollados o no; podemos abrirnos a la diversidad sexual; jóvenes, o no tan jóvenes; vivir con una "discapacidad", tal y como afirma María José Guerra Palmero, filósofa y feminista española (Muñiz Pérez, 2018). La interseccionalidad consiste en valorar la conjunción de las diferentes opresiones y ésta es una de las palancas que mueve a la teoría feminista a dar respuesta a las injusticias, una manera de enfrentar políticamente las desigualdades y discriminaciones para aspirar a vivir en sociedades decentes y justas.

Desafortunadamente la crisis climática aumenta la presión sobre los grupos marginados y precarizados incrementando las brechas sociales por inequidad de acceso a recursos básicos como la alimentación. La inseguridad alimentaria y nutricional son consecuencias graves del cambio climático que afectan directamente 
la salud de las mujeres, quienes lidian con el cuidado y la manutención de los hijos $y$, en ocasiones, de la familia extendida ante la escasez de recursos finitos.

El cambio climático empeorará las condiciones de vida de agricultores, ${ }^{5}$ pescadores y quienes viven de los bosques, poblaciones ya de por sí vulnerables y en condiciones de inseguridad alimentaria. Aumentarán el hambre y la malnutrición. Las comunidades rurales, especialmente las que viven en ambientes frágiles, se enfrentan a un riesgo inmediato y creciente de pérdida de las cosechas y del ganado, así como a la reducida disponibilidad de productos marinos, forestales y provenientes de la acuicultura. Los episodios climáticos extremos, cada vez más frecuentes e intensos, tendrán un impacto negativo en la disponibilidad de alimentos, el acceso a los mismos, su estabilidad y su utilización, así como en los bienes y oportunidades de los medios de vida tanto en zonas rurales como urbanas. La población empobrecida correrá el riesgo de inseguridad alimentaria por la pérdida de sus bienes. La capacidad de la población rural de convivir con los impactos producidos por el cambio climático, depende del contexto cultural y de las políticas existentes, así como de factores socioeconómicos como el género, la composición de los hogares, la edad y la distribución de los bienes en el hogar (FAO et al., 2018: 37-112).

El cambio climático agravará las inequidades que ya viven las mujeres en situación de pobreza. Las mujeres indígenas, ${ }^{6}$ afrodescendientes y migrantes son más vulnerables que otras mujeres en el mundo, ya que carecen de acceso a servicios de salud en general, por ende, no cuentan con atención y educación en salud sexual y reproductiva, ni acceso al aborto incausado. Los países en vías de desarrollo tienen más restricciones para interrumpir el embarazo, a diferencia de la mayoría de los países desarrollados que permiten el aborto incausado (Vogelstein y Turkington, 2019: 2). Estas políticas privan a las mujeres que viven en países subdesarrollados del acceso a una reproducción justa, a la libertad de elegir si desean o no tener hijos bajo las condiciones en que viven $y$, esencialmente, del derecho fundamental a la salud.

El concepto de interseccionalidad lo desarrolló Kimberlé Crenshaw en 1989 para entender cómo los aspectos sociales y de identidad política de una persona pueden crear formas de discriminación o privilegio, ya sea por su aspecto físico, el

${ }^{5}$ Las tasas de suicidio sin precedentes entre agricultores indios en los últimos años se han relacionado con los efectos nocivos en la producción agrícola de subsistencia debido al aumento de la temperatura por el cambio climático. Dejando a las mujeres con mayores cargas de trabajo y empeorando su condición de pobreza (Murari, Jayaraman y Swaminathan, 2017).

${ }^{6}$ Las mujeres indígenas mexicanas soportan la mayor carga y ofrecen la mayor resistencia ante las injusticias ambientales (Gaard y Opperman, 2013: 10). 
estrato social, el origen étnico, la cultura, la sexualidad, el género, e incluso sus habilidades. Es decir, estas características se intersecan y suman, creando así fenómenos de privilegio y de vulnerabilidad. De ahí que una mujer blanca y una mujer negra tengan una vulnerabilidad distinta.

La perspectiva de interseccionalidad nos ayuda a identificar cómo los sistemas de poder afectan más a los excluidos sociales, y sirve como marco de referencia para visibilizar la necesidad de la justicia reproductiva frente al cambio climático. Las mujeres más afectadas por la crisis climática y la ausencia de políticas reproductivas habitan en África Subsahariana, Asia Meridional y América Latina. En este sentido, la incorporación de la perspectiva de la interseccionalidad en el análisis pondría en la agenda temas importantes como la libertad reproductiva ${ }^{7}$ y la justicia, no sólo reproductiva sino ambiental ${ }^{8}$ para las mujeres racializadas y en condición de pobreza en el mundo.

Una nueva visión de justicia reproductiva fue planteada por Loretta Ross a finales de los años noventa, que implicaba la unión de los derechos reproductivos y la justicia social junto a tres principios primarios: 1) el derecho a no tener un hijo, 2) el derecho a tener un hijo y 3 ) el derecho a criar hijos en un ambiente saludable. La justicia reproductiva demanda autonomía sexual y libertad de género. Para lograrlo, es indispensable el acceso a bienes específicos comunitarios que incluyan un sistema sanitario de alta calidad, vivienda, educación, salario digno, un ambiente saludable y una red de seguridad en caso de que estos recursos fallen. El manejo seguro y digno de la fertilidad, el nacimiento y los cuidados parentales son imposibles sin estos recursos (Ross y Solinger, 2017: 9). Esta perspectiva de justicia reproductiva surgió porque el paradigma dominante no atendía las necesidades de las mujeres marginadas socialmente, en particular las necesidades de las mujeres negras en Estados Unidos para ejercer decisiones reproductivas como sus contrapartes más privilegiadas que vivían, y siguen viviendo, en mejores condiciones para enfrentar dichas decisiones, a partir de un ambiente más seguro, saludable y con acceso a recursos que superan lo indispensable.

${ }^{7}$ Que implica la conciencia de sí mismo, establecer un plan de vida, estabilidad emocional, la oportunidad de elegir, la garantía de los derechos reproductivos y el acceso a una vida saludable y proactiva.

${ }^{8}$ La justicia ambiental entendida como el trato justo y la participación significativa de todas las personas, independientemente de su raza, color, origen nacional o ingresos, con respecto al desarrollo, implementación y cumplimiento de las leyes, regulaciones y políticas ambientales definida por la Agencia de Protección Ambiental de los Estados Unidos. (https://www.epa.gov/environmentaljustice). 


\section{Autonomía, vulnerabilidad y el principio de igualdad de trato y oportunidades}

La vulnerabilidad que sufren las mujeres más pobres alrededor del mundo ante la crisis climática deteriora su autonomía. Lizbeth Sagols propone en La ética ante la crisis ecológica, una autonomía que vaya más allá del antropocentrismo, una autonomía que nos debemos unos a otros, consciente de la comunidad y fuente del reconocimiento de nuestra continuidad y vínculo con todo lo vivo (Sagols, 2006: 114). Necesitamos apelar a una conciencia ética, basada en la admiración y el respeto a todo lo vivo y al conjunto de la vida en sí. Sagols parte del concepto de O. Wilson y de Erich Fromm para proponer que la ética actual ha de tener como eje la biofilia, el amor a lo viviente. La ética actual ha de comprender que mujeres, hombres, otros seres vivos y ecosistemas formamos parte de la vida; por tanto, ha de construirse desde una auténtica ecoética y reconocer la unidad e igualdad básica de valor de todo lo que vive (Sagols, 2014: 26). Desde este eje podemos abordar la condición vulnerable que lastima directamente a poblaciones marginadas de mujeres y niños, así como al ambiente, y que apoye la toma de decisiones reproductivas, sin tomar en cuenta solamente el proyecto de vida personal o la condición en la que se vive, sino principalmente el impacto del nacimiento de un hijo y su interacción con el medio ambiente y las otras formas vida que componen el ecosistema al cual habrá de pertenecer.

Las mujeres hoy en día deben enfrentarse a mecanismos de política pública que no les permite ejercer su autonomía y libertad reproductiva; tienen hijos en condiciones precarias y enfrentan las dificultades sociales, el desastre ecológico y económico ocasionado por la crisis climática, y por otras catástrofes, como la pandemia que sufrimos actualmente por el virus SARS-CoV-2, ${ }^{9}$ que empeora las vulnerabilidades de las mujeres precarizadas y las expone a mayor violencia doméstica y embarazos no deseados. Florencia Luna utiliza la metáfora de las capas para concebir el concepto de vulnerabilidad, y pone de ejemplo a las mujeres provenientes de países industrializados que son respetadas, pueden estudiar, trabajar y elegir su plan de vida. En cambio, las mujeres que viven en países intolerantes a los derechos reproductivos adquieren una primera capa de vulnerabilidad. Aunque menciona que la situación cambia si la mujer cuenta con educación y recursos, pues

\footnotetext{
${ }^{9}$ La pandemia de este virus inició en Wuhan China en diciembre de 2019 en un mercado húmedo debido al comercio y consumo de animales silvestres, facilitando el "salto" del virus de animales a seres humanos. La prohibición de los mercados debe ser de carácter global, asimismo las prácticas de consumo de animales silvestres. Es indispensable después de esta experiencia implementar regulaciones más estrictas para evitar la zoonosis y la posibilidad de nuevas pandemias en el futuro por esta misma causa.
} 
puede superar algunas de las consecuencias de la intolerancia a los derechos reproductivos. Pero una mujer pobre, viviendo en un país intolerante a los derechos reproductivos, adquiere otra capa de vulnerabilidad. Y así se van añadiendo capas sucesivamente, y éstas se manifestarán de diversas formas de acuerdo con su condición y contexto (Luna, 2019: 88).

Por otro lado, ser vulnerable es una característica universal de cualquier organismo, vista como potencialidad, fragilidad, y no estado de dańo. Esa vulnerabilidad puede ser disminuida respetando los derechos humanos básicos en un orden social justo al que se puede aspirar con políticas de mitigación, en las que se integre la perspectiva de interseccionalidad, justicia reproductiva y transversalidad de género, tendiendo a la protección equitativa de los individuos contra daños para impedir que su vulnerabilidad sea transformada en una lesión a su integridad. El ser vulnerado se refiere a una situación de hecho, de daño actual, que tiene consecuencias relevantes al momento de la toma de decisiones. Atendiendo a los daños sufridos, las vulneraciones requieren cuidados especiales por parte de los Estados y las instituciones sociales organizadas. Es decir, éstas deben instalar servicios terapéuticos y de protección, como servicios sanitarios, asistenciales, educacionales, etcétera, para disminuir y remover daños con el fin de que los desfavorecidos se empoderen (de Morais y Monteiro, 2017: 314). Esto requiere por parte de los Estados participantes en la mitigación en contra del cambio climático de acciones afirmativas y reparadoras que influyan en la autonomía, la integridad y la dignidad de las poblaciones vulneradas.

La transversalidad de género busca promover la equidad por medio de la construcción de capacidades y la responsabilidad de género, así como la planificación de las políticas públicas teniendo en cuenta las desigualdades existentes, la identificación y la evaluación de los resultados e impactos producidos por éstas. La transversalidad de género aplica a las políticas públicas el principio de igualdad de trato y de oportunidades entre mujeres y hombres. De esta manera se garantiza el acceso a todos los recursos en igualdad de condiciones. La integración de este principio implica al menos dos tipos de cambio en el modelo de gestión: el primero, es el aspecto procedimental, poniendo en el centro de las políticas la diferente situación y posición social de mujeres y hombres con el objetivo de satisfacer de forma equitativa las necesidades de ambos sexos; el segundo va dirigido a revisar las estructuras y formas de organización de la administración pública para erradicar, desde su base, los elementos estructurales que hacen que se mantengan las desigualdades sociales entre ambos sexos (Gobierno de Navarra, 2018). Este principio permitiría promover la justicia y libertad reproductiva desde un marco más equilibrado de acceso a recursos y bienes, una gestión ante la crisis climática en la que 
las mujeres más vulnerables puedan ejercer su autonomía bajo condiciones justas, y éstas sirvan de contrapeso ante la vulnerabilidad que viven por el problema de injusticia y pobreza.

\section{Política interseccional, justicia reproductiva y las desigualdades socioecológicas}

Giovanna Di Chiro sostiene que la política interseccional de la justicia reproductiva ha vinculado los derechos a la autodeterminación corporal y el derecho a opciones seguras de anticoncepción y aborto (el derecho a no tener hijos), con el derecho a tener hijos — lo que implicaría tener acceso a la reproducción asistida—y a poder criarlos de manera nutritiva, saludable y en ambientes seguros. La justicia reproductiva expone una amplia gama de problemas sociales, económicos y ambientales que deben verse como problemas reproductivos significativos e incluir en los programas empleos de calidad y seguridad económica, libertad contra la violencia doméstica y la esterilización forzada, atención médica asequible, oportunidades educativas, viviendas dignas y acceso a vecindarios limpios y saludables. Este desafío del movimiento de justicia reproductiva al discurso dominante de los derechos reproductivos como derechos de aborto apunta a la importancia de la lucha para lograr la reproducción social para las mujeres pobres y las mujeres de color (Di Chiro, 2009: 2).

Aproximadamente, el $80 \%$ de la población mundial (el sur global) ha generado alrededor del $20 \%$ de las emisiones de gases de efecto invernadero (GEI); en otras palabras, el otro $20 \%$ de la población (el norte global) es responsable del $80 \%$ de las emisiones de GEI en nuestra atmósfera. A pesar de la claridad de esta lógica, el argumento de la sobrepoblación reapareció en las publicaciones de la reunión sobre Cambio Climático de las Naciones Unidas en Copenhague en el año 2009, con propuestas para la planificación familiar entre las comunidades pobres como un método costo-efectividad para reducir las emisiones de carbono. La organización sin fines de lucro UK Population Matters lanzó un sistema de "compensación de población" similar a los sistemas de compensación de carbono, disponible para su compra por el jet set de los consumidores del primer mundo. Ninguna de estas estrategias sugiere la reducción del consumo alarmante del primer mundo de los recursos del planeta, o la restricción de su contribución con la emisión del $80 \%$ de los GEI. Reducir una tercera parte de la población mundial se convierte en algo importante cuando los superconsumidores del primer mundo se dan cuenta que los resultados más severos que ya golpean a la parte más marginalizada 
del mundo crearán una crisis de refugiados, una migración urgente para la gente pobre. Además, estos países perciben la migración como una amenaza, y los refugiados por cambio climático han inspirado argumentos para el incremento de la militarización como protección en contra de la migración. Habrá que mirar hacia ambos lados, reconocer las intersecciones entre la justicia climática y la justicia reproductiva, afirma Greta Gaard (2017: 126-130).

Distintos análisis geográficos sobre el impacto climático sugieren la probabilidad de consecuencias dramáticamente diferentes para las poblaciones alrededor del mundo. Al examinar críticamente las relaciones socioecológicas, se observa con claridad la responsabilidad y vulnerabilidad desproporcionada (Barclay, 2020: 10). La distribución desigual de los riesgos y de los costos ambientales no tiene neutralidad de género; son el resultado de estructuras, regulaciones y procesos de negociación política y económica. Debido a la globalización, los riesgos y los costos ambientales son crecientemente deslocalizados y trasladados a regiones menos poderosas y a grupos sociales más vulnerables (Boatcă, 2011).

La crisis climática obliga a las comunidades más afectadas de los países del sur global a migrar a los países desarrollados, exponiendo su vida y naufragando en los océanos, o muriendo al atravesar los desiertos, suscitando así un exilio por cambio climático, una crisis migratoria, mientras que los países desarrollados responsables de la mayor emisión de GEI son incapaces de enfrentar la crisis respetando los derechos fundamentales de los refugiados por cambio climático, muchos de los cuales son mujeres.

La expresión "racismo ambiental" fue acuñada por Benjamin F. Chavis, la cual refiere a la disminución racial en la elaboración de políticas ambientales, la desigual ejecución de las leyes y regulaciones ambientales y la deliberada selección de comunidades de color para ubicarlas en lugares con desechos tóxicos y contaminantes. Algunos estudios realizados en la década de los ochenta y los primeros ańos de los noventa encontraron que tanto la raza como el ingreso eran variables específicas importantes ya que por separado o conjuntamente explicaban las desigualdades ambientales. Además, estudios realizados por economistas en los ańos setenta encontraron que los pobres también sufrían una alta proporción de los costos ambientales (costos estructurales regulatorios distribuidos decrecientemente), y recibían menos proporción de los beneficios ambientales (Driscoll de Alvarado y Márquez-Padilla, 2001: 289).

Las activistas de la justicia reproductiva creen que este marco alentará a más mujeres de color y a otros grupos marginados a involucrarse más en movimientos políticos para promover la libertad reproductiva. La meta de la justicia reproductiva es transformar la manera en la que todos conceptualizamos y entendemos la libertad reproductiva (Price, 2010: 43-61). Afortunadamente, una serie de aca- 
démicas de la justicia ambiental, la justicia sexual y la justicia reproductiva, han expuesto el imperialismo, racismo, sexismo y heterosexismo inherentes a las plataformas de eugenesia ambiental, al igual que el equivocado enfoque de la reproducción como la fuente primordial del daño ambiental. Los defensores de la justicia ambiental y la justicia sexual y reproductiva señalan que las políticas ambientales eugenésicas han afectado los derechos sexuales y reproductivos de las mujeres al imponer métodos peligrosos y coercitivos de control de natalidad y la esterilización involuntaria de mujeres negras e indígenas en el sur global y en Estados Unidos, en nombre de la protección ambiental (Stein, 2013: 187).

\section{Conclusiones}

Necesitamos abordar la discusión, el análisis y la elaboración de políticas públicas frente al cambio climático bajo la lente de interseccionalidad y de justicia reproductiva, el principio de igualdad de trato y oportunidades, y una perspectiva ética bajo el eje de la biofilia. Necesitamos mitigar el daño que se ha venido ocasionando a las mujeres más marginadas y afectadas por la crisis climática, colocarlas en el centro de los planes hacia el futuro, fomentar su empoderamiento a través de una economía que les permita acceder a la propiedad de la tierra, ya que sólo represen$\tan$ un $13 \%$ de los propietarios de tierra en el planeta. También es importante brindarles acceso a efectivo y crédito, educación superior que les permita la paridad de género, salud en general que garantice el acceso a salud sexual y reproductiva, un ambiente limpio y seguro para vivir y disminuir su vulnerabilidad ante los efectos de los cambios ambientales, de los cuales no sean capaces de recuperarse, ampliando así la brecha de género en la agricultura.

\section{Referencias bibliográficas}

Bонтӑ, Manuela. (2011). "Global Inequalities Transnational Processes and Transregional Entanglements" desiguALdades.net, Research Network on Independent Inequalities in Latin America, Working Paper Series, (11).

Butterbaugh, Laura. (2005). "Why did Hurricane Katrina hit women so hard?". Off Our Backs, 35(9/10), 17-19.

Collomb, Jean-Daniel. (2014). "The ideology of climate change denial in the United States". European Journal of American Studies, 9(1), 1-17. https://doi. org/10.4000/ejas.10305. 
De Morais, Talita Cavalcante Arruda; Monteiro, Pedro Sadi. (2017). "Los conceptos de vulnerabilidad humana y la integridad individual para la bioética”. Revista Bioética, 25(2), 311-319. http://dx.doi.org/10.1590/198380422017252191.

de Vengoechea, Alejandra. (2012). Las cumbres de las Naciones Unidas sobre Cambio Climático. Colombia: Proyecto Energía y Clima de la Fundación Friedrich Ebert. Recuperado de https://library.fes.de/pdf-files/bueros/la-energiayclima/09155.pdf.

Di Chiro, Giovanna. (2009). "Sustaining everyday life: bringing together environmental, climate and reproductive justice". Different Takes (Climate Change Series), (58), https://www.srhr-ask-us.org/publication/sustaining-everyday-lifebringing-together-environmental-climate-reproductive-justice/.

Driscoll de Alvarado, Bárbara; Márquez-Padilla, Paz Consuelo (coords.). (2001). El color de la tierra. Las minorías en México y Estados Unidos. México: UNAM, CISAN.

FAO; IFAD; UNICEF; WFP; WHO. (2018). The State of Food Security and Nutrition in the World 2018. Building climate resilience for food security and nutrition. Roma: FAO. Recuperado de http://www.fao.org/3/I9553EN/i9553en.pdf.

GaARD, Greta. (2017). Critical ecofeminism. Nueva York: Lexington Books.

GaArd, Greta; Oppermann, Serpil. (2013). International perspective in feminist ecocriticism. Nueva York: Routledge; Taylor \& Francis.

Gobierno de Navarra. (2018). Estrategia para la participación social y politica de las mujeres en las políticas públicas de la Comunidad Foral de Navarra 2018-2020. Navarra: Instituto Navarro para la Igualdad. Recuperado de http://www.navarra.es/NR/rdonlyres/9853FBF1-064F-481D-9DDC-AA4CC13CAFC9 /432815/Estrategia.pdf.

Grupo de Información en Reproducción Elegida, A.C. (2018). La pieza faltante. Justicia reproductiva. Ciudad de México: Autor. Recuperado de https:// justiciareproductiva.gire.org.mx/assets/pdf/JusticiaReproductiva.pdf.

Haines, Andy; Ebi, Kristie. (2019). "The Imperative for Climate Action to Protect Health". The New England Journal of Medicine, (380), 263-273. http://doi. org/10.1056/NEJMra1807873.

LunA, Florencia. (2019). "Identifying and evaluating layers of vulnerability-a way forward”. Developing World Bioethics, 19(2), 86-95. https://doi.org/10.1111/ dewb.12206.

Muñiz Pérez, Tamara. (2018, 15 de febrero). "El ecofeminismo plantea un cambio de conciencia, pensarnos más allá del especismo” (en línea). Pikara Online 
Magazine; En red. Recuperado de https://www.pikaramagazine.com/2018/02/ maria-jose-guerra-palmero/.

Murari, Kamal Kumar; Jayaraman, T.; Swaminathan, Madhura. (2017). "Climate change and agricultural suicides in India". Proceedings of the National Academy of Sciences of the United States of America, 115(2), e115. https://doi. org/10.1073/pnas.1714747115.

ONU Mujeres. (2014 [1995]). Declaración política y documentos resultados de Beijing+5. Naciones Unidas. Recuperado de https://www.unwomen.org/es/ digital-library/publications/2015/01/beijing-declaration.

Price, Kimala. (2010). "What is Reproductive Justice?: how women of color activists are redefining the Pro-Choice paradigm". Meridians, 10(2), 42-65. https:// doi.org/10.2979/meridians.10.2979/meridians.2010.10.2.42.

Ross, Loretta; Solinger, Rickie. (2017). Reproductive Justice. An introduction. Oakland, CA: California University Press.

SAGols, Lizbeth. (2006). Interfaz bioética. México: Fontamara; UNAM.

SAGols, Lizbeth. (2014). La ética ante la crisis ecológica. México: Fontamara; UNAM. STEIN, Rachel. (2013). "Sex, population, and environmental eugenics in Margaret Atwood's Oryx and Crake and The Year of the Flood". En Greta Gaard, Simon C. Estok y Serpil Opperman (eds.), International Perspective in Feminist Ecocriticism. Nueva York: Routledge; Taylor \& Francis. 184-202.

Vogelstein, Rachel B.; Turkington, Rebecca. (2019). "Abortion law: global comparisons" (en línea). Council on Foreign Relations. Women and Foreign Policy Program. Recuperado de https://www.cfr.org/article/abortion-law-globalcomparisons. 